\title{
SANDS5.0984C \\ Observation of Refractive Index Gradients $951146--3$ \\ in a Laser-Generated Plume \\ Using Laser Schlieren Imaging
}

\author{
M. Essien, D. M. Keicher, and J . L. Jellison \\ Sandia National Laboratories \\ Albuquerque, New Mexico 87185-1411
}

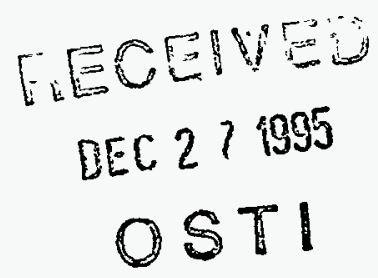

\begin{abstract}
Understanding the interaction between a pulsed Nd:YAG laser beam and the plume generated above the surface of a workpiece during laser welding is important for process modeling and control. We present a laser schlieren method which can be used to observe this interaction by imaging the refractive index gradient within the plume. The laser schlieren technique uses an expanded, collimated probe laser beam to image a phase object onto a filter with a radial gradient in optical density. The probe beam is focused through the filter and onto the image plane of an intensified high-speed camera, so that the temporal variation of the laser/plume interaction may be observed over the duration of the laser pulse. Qualitative measurements of the spatial distribution of refractive index are presented. The velocity of the vaporized metal forming the plume is also measured for a stainless steel weldment.
\end{abstract}

\section{Introduction}

The process of laser welding of metals invariably produces a plume of vaporized metal atoms and ions above the surface of the workpiece. Quantitative study of this plume is essential for process monitoring and control, and is also needed to provide input for computational models of laser welding. Indeed, characterization of the physical properties of the plume, i.e., size, velocity, and morphology, is necessary in order to determine the intensity distribution at the surface of the workpiece. The physical properties of the plume will determine the extent of its interaction with the laser radiation. Figure 1 shows a series of high-speed photographs of the plume generated above the surface of a stainless steel workpiece. The incident laser radiation is from a pulsed $\mathrm{Nd}$ :YAG laser, with $\lambda=1.06 \mu \mathrm{m}$, delivering $0.44 \mathrm{MW} / \mathrm{cm}^{2}$ onto the metal surface. Some crucial questions that arise from these observations are: (1) Is the plume dense enough to cause significant refraction of the laser radiation? (2) How can this interaction be monitored in real time?, (3) What is the morphology of the plume, and (4) How does this morphology evolve as the laser pulse evolves? The first question may be addressed by either studying the weld pool or by observing the plume. Using the former method, and alloys with very volatile components, it is possible to extract qualitative information about the beam/plume interaction. Indeed, surface active elements can play a significant role in the laser welding processes. ${ }^{1,2}$ For materials such as 304 stainless steel, a low sulfur content can lead to a wide, shallow weld, due to an outward flow of the molten material. Addition of sulfur, can give an extremely different result. For example, in a material with a high surface active element content, such as sulfur, flow of the liquid metal in the melt region tends to be in the direction of an increasing thermal gradient, or

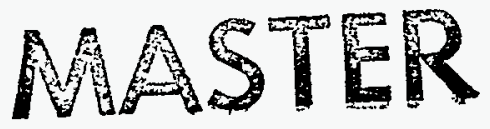


towards the center of the weld pool, creating a deeper weld. This type of flow may yield qualitative information about the intensity distribution at the metal surface. The second, third, and fourth questions may be addressed by using a laser schlieren technique to observe refraction of a probe laser by the plume.
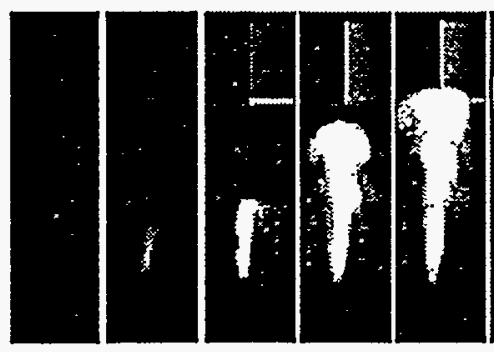

0.50

0.60
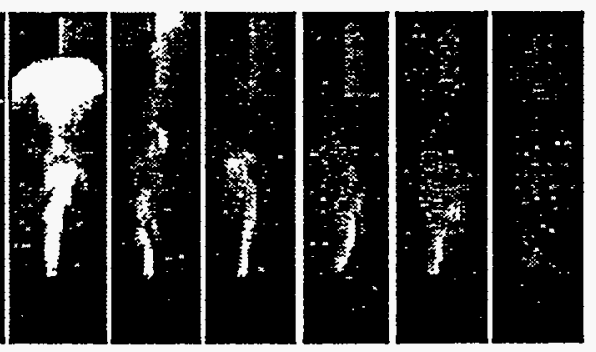

$3.16 \quad 3.83$

Figure 1: High-speed camera images of the plume generated by focusing $1.06 \mu \mathrm{m} \mathrm{Nd:YAG} \mathrm{radiation} \mathrm{onto} 304$ stainless steel. The intensity delivered to the metal surface was $0.44 \mathrm{MWatts} / \mathrm{cm}^{2}$. The times shown for each frame are in milliseconds and are measured with respect to the laser pulse.

Cieslak and Jellison ${ }^{1}$ have studied the effects of sulfur, a surface active element, in convection mode laser welds on 304 stainless steel, using a pulsed Nd:YAG laser. The results observed contradict those expected due to the preceding explanation of flow behavior with and without surface active elements. Figure 2 shows metallographic cross-sections of single spot welds produced on 304 stainless with a high sulfur content. Each of these welds was produced using the same laser parameters, with and without the laser plume present. As seen from these photographs, there is a small increase in penetration for the weld produced without the plume present. This result may suggest that the vapor plume has some effect on the laser beam. Similar single spot welds were produced on a low sulfur stainless steel, Figure 3. Again the plume was present for one weld and removed in the case of the second weld. In this case (low sulfur) the plume effect became more pronounced. When the plume was present a significant increase in penetration occurred, compared to the weld produced with the plume removed from the surface. The very broad, shallow weld produced without the plume is typical of a convection mode weld in a material without a significant surface active alloying element. However, the deeper penetration achieved in the low sulfur material in the presence of the plume provided an unexpected result. 


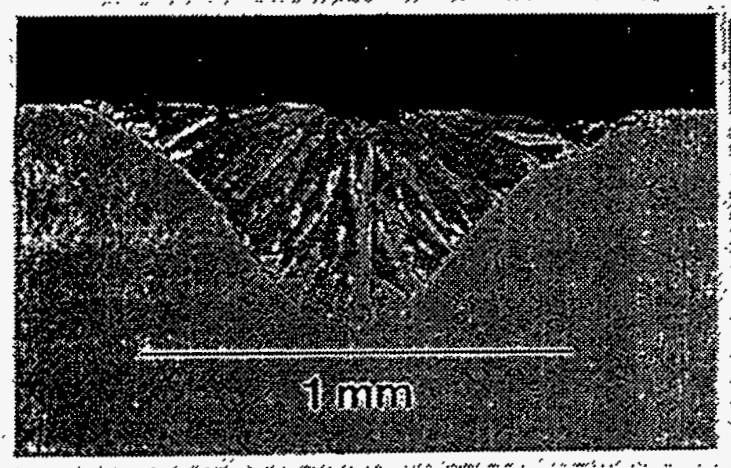

a

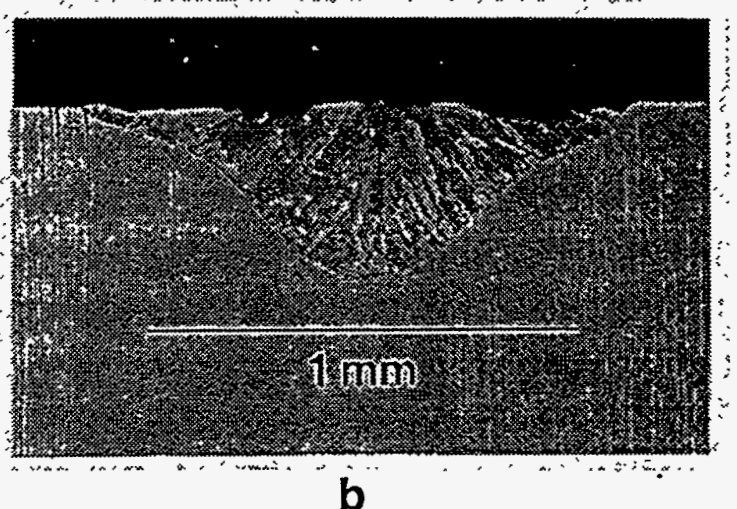

b

Figure 2: Micrographs of spot weld of high-sulfur 304 stainless steel ( 0.05 wt. \% S: (a) without transverse Ar flow, (b) with transverse Ar flow. For both welds, the pulse width is $5.7 \mathrm{~ms}$. The pulse energy is 17.3 J/pulse. From Cieslak, M. J., and Jellison, J. L. (1989). A Perspective on Welding Science. MRS Bulletin, XIV, 2, 32-38.

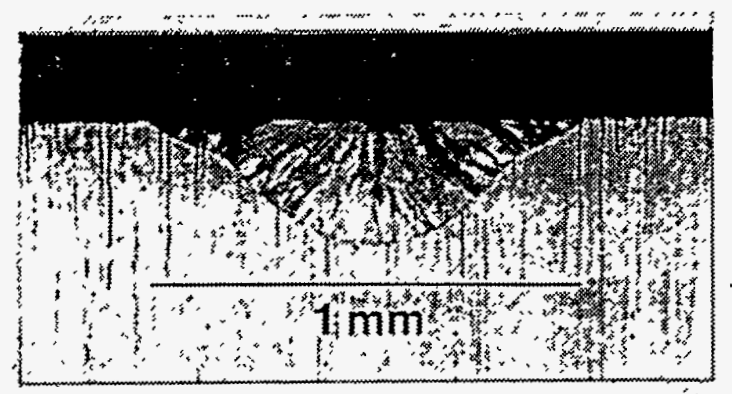

a

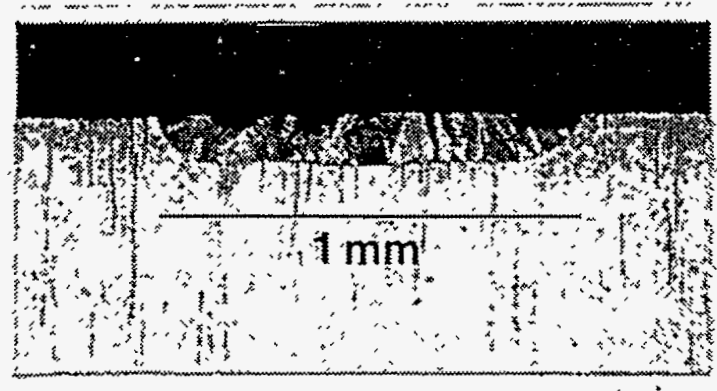

b

Figure 3: Micrographs of spot weld of low-sulfur 304 stainless steel (0.005 wt. \% S: (a)without transverse Ar flow, (b) with transverse Ar flow. For both welds, the pulse width is $5.0 \mathrm{~ms}$. The pulse energy is 9.8 J/pulse. From Cieslak, M. J., and Jellison, J. L. (1989). A Perspective on Welding Science. MRS Bulletin, XIV, 2, 32-38.

\section{Experiment}

From these results, it was obvious that the laser plume can play an important role in determining the weld characteristics. To identify the phenomenon which would explain this change, several experiments were performed to analyze the plume. ${ }^{3}$ Time-resolved scattering measurements showed that some scattering would occur, however, not until very late into the pulse. In situ, time-resolved laser absorption measurements showed that the absorption of the 
laser energy into the work piece during a single pulse remained nearly constant once equilibrium was established. Finally, spectroscopic analysis and electron density measurements revealed that electron densities are insufficient to infer the existense of a plasma. High-speed film images of the plume showed a very erratic behavior, even during the short 5-7 ms pulses from the Nd:YAG laser. Putting the various data together provides a deeper understanding of the laser welding process. In summary, ionization was insufficient to produce a plasma, scattering was not significant, and the laser absorption was essentially constant. Yet the extent of the plume varied significantly with time. This supports the premise that a thermal gradient generated by the plume causes the beam to be defocused. This defocusing would reduce the beam intensity at the weld surface, causing vaporization to be reduced. With this reduced vaporization, the beam would again be sharply focused onto the weld specimen, leading to increased vaporization. This process would go in a cyclic fashion.

\section{Beam/Plume Studies}

To evaluate this concept, we first generated a model to simulate the beam/plume interaction. A cylindrically symmetric refractive index gradient was synthesized, and a Gaussian beam was mathematically propagated through this medium. The results indicated that the refractive index gradient would cause the Gaussian beam to defocus into an annulus whose peak intensity was significantly reduced. ${ }^{4}$ Because of this annular distribution, the peak intensity of the beam was shifted away from the center of the weld pool. This would explain the higher penetration for the low sulfur material in the presence of the plume since, if an annular beam distribution occurs during laser welding, the flow for the low sulfur material would be away from the thermal gradient, or in this case, towards the center of the weld pool.

Next an axicon optical element was used to produce this annular beam distribution at the metal surface, Figure 4.

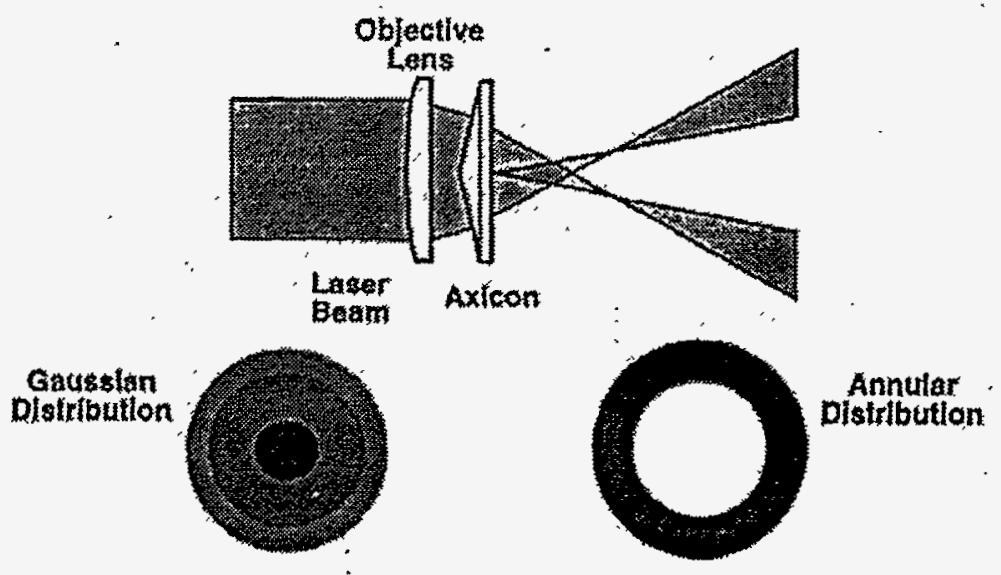

Figure 4: $\quad$ Schematic of axicon lens used to create an annular intensity distributiọn. 
For all of these tests the plume was removed from the surface. A series of welds were made at different focus positions and powers on both high sulfur and low sulfur materials. Metallographic cross-sections of two of these welds made at the same laser conditions are shown in Figure 5. Figure $5 \mathrm{~b}$ shows the weld made on the high sulfur material. As expected, the deepest penetration occurs within the annular region. It is also obvious from the figure that very little melting has occurred at the center of the weld. Conversely, for the low sulfur material, the maximum penetration occurs at the center of the weld. For this particular welding condition, there is very little laser energy at the center of the weld region. These results demonstrate in a qualitative fashion, that a refractive index gradient produced by the plume would indeed explain the reversal in the flow behavior observed in the presence of the laser plume.

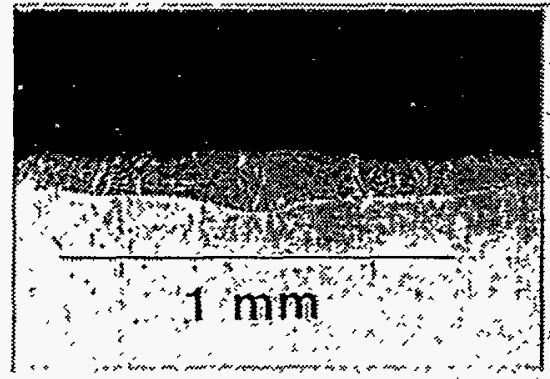

0.001 wt. \% sulfur

a
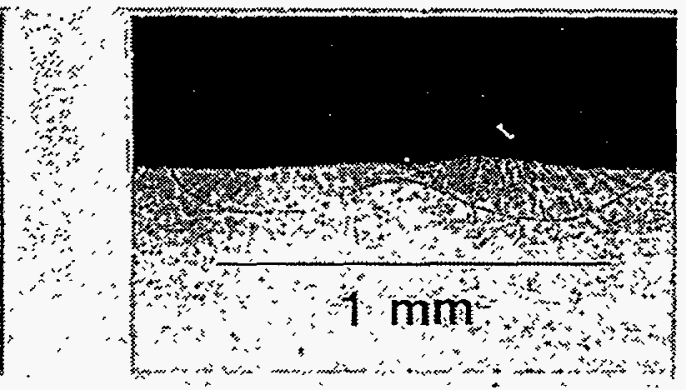

0.022 wt. \% sulfur

b

Figure 5: Micrographs of spot weld of low-sulfur and high-sulfur 304 stainless steel made using an axicon lens. (a) 0.001 wt \% sulfur. (b) 0.022 wt. \% S.

\section{Laser Schlieren Imaging}

Schlieren imaging is a technique that allows direct observation of refractive index gradients within a transparent object. 5 In this technique a collimated light source (helium-neon laser) is sent through the plume and is deviated due to the refractive index gradients within the plume. The probe beam is then decollimated and focused onto a schlieren filter. The filter used in this work has a diameter of $6.3 \mathrm{~mm}$ and was computer generated and recorded on slide film. The developed slide serves as the actual filter. A schematic of the essential elements of the laser schlieren system is shown in Figure 6. The probe laser is expanded and focused onto the pinhole of a spatial filter. The pinhole typically has a diameter of $20-50 \mu \mathrm{m}$ and determines the spatial resolution of the final schlieren image. The probe is then collimated by lens 1 . The region between lens 1 and lens 2 (decollimator), is the test section. If a phase object (an object whose refractive index has no imaginary part) is placed within the test section, any nonuniformity in refractive index will cause a light ray to be deflected from the collimated light path. The ray 
crosses the test section and is focused onto the schlieren filter by lens 2 . The schlieren filter replaces the knife edge filter of the conventional schlieren technique, ${ }^{6}$ so that rays deflected by nonuniformities in a transparent medium are imaged onto regions of the filter with reduced transmission. Therefore lines of constant intensity in the schlieren image represent constant deflection. An image of the filter used in this work is shown in Figure $6 \mathrm{~b}$.

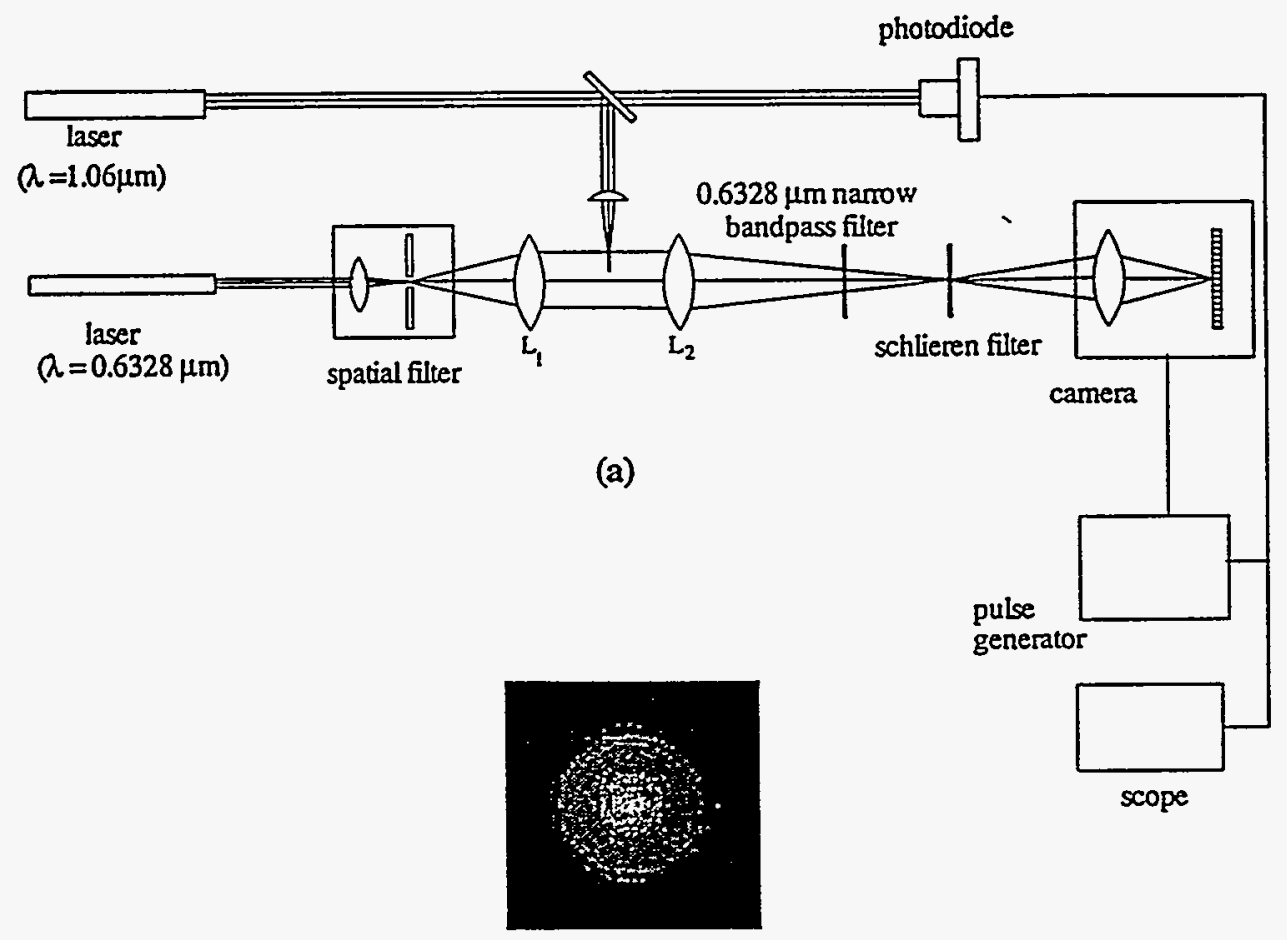

(b)

Figure 6: (a) Schematic of laser schlieren apparatus. (b) Laser schlieren filter.

The filter has a linear gradient in opacity that proceeds outward radially from the center, so that an undeviated ray passes through the filter and suffers little or no attenuation, while rays that are deviated from the axis of the system are focused onto non-transparent regions of the filter. Therefore a region of the plume that causes large deviations will appear dark when imaged through the schlieren filter.

The evolution of the plume is recorded using a Kodak Ektapro high-speed camera operating at a rate of 6000 frames per second, with an exposure time of $10 \mu \mathrm{s}$. A Raytheon SS 501 pulsed $\mathrm{Nd}$ :YAG serves as the processing laser. The radiation is focused onto the surface of a 304 stainless steel target by a 4 inch plano-convex, fused silica lens. A photodetector senses the laser pulse and sends a signal to a pulse generator (Stanford Research Systems, 535) 
and to an oscilloscope. The pulse generator then sends a signal to the high-speed camera, triggering a recording of the plume.

\section{Results}

Figure 7 shows the images of the probe laser as it propagates through the test section during a pulse of the Nd:YAG processing laser incident on 304 stainless steel. In each case the processing laser propagates from top to bottom and is focused onto the metal, which appears as a dark, rectangular region at the bottom of the frame. The laser operates at 0.3 joules per pulse and is focused to a spotsize radius of $200 \mu \mathrm{m}$. This corresponds to an intensity of $0.05 \mathrm{MW} / \mathrm{cm}^{2}$. The times marking each frame are in milliseconds and are measured with respect to the arrival of the laser pulse. Figure 7 shows that the plume propagates away from the surface of the metal in the form of a highly refractive, lever-shaped region of gas. Refraction of the probe laser by the plume is clearly visible as well-defined dark regions that follow the contours of the plume. This is clearly visible in Figure 7 at $1.00 \mathrm{~ms}$. This type of geometry is maintained past $2.50 \mathrm{~ms}$, after which turbulent mixing with the ambient gas begins. Figure 7 shows that light rays propagating through the central regions of the plume do not suffer significant refraction. However, as rays strike the plume boundary further from the plume axis, curvature effects begin to refract the rays onto dark regions of the filter. An intermediate region near the edge of the plume exists were the curvature of the plume causes rays to emerge undeviated, and focused through the center of the filter. At the edge of the plume, light rays are again refracted, and deviated onto dark regions of the filter. Figure 8 shows the evolution of the plume for the case of a pulse energy of 5.1 joules, corresponding to an intensity of $0.90 \mathrm{MW} / \mathrm{cm}^{2}$. In this case, significant refraction by the plume is evident after only $0.83 \mathrm{~ms}$. Turbulent flow is reached around $1.3 \mathrm{~ms}$.
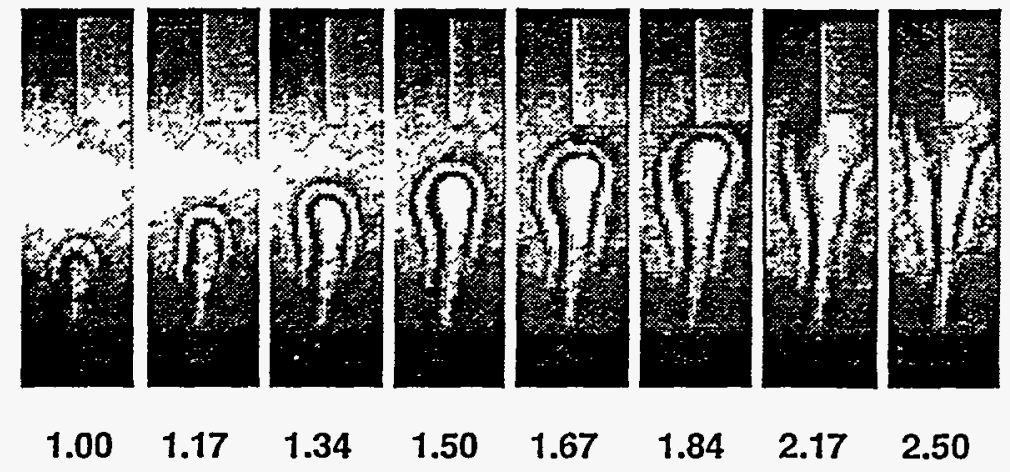

Figure 7: High-speed laser schlieren images of the plume generated above 304 stainless for an intensity of $0.05 \mathrm{MW} / \mathrm{cm}^{2}$. The times shown for each frame are in milliseconds.

The position of the plume from about 0.6 to $1.6 \mathrm{~ms}$ is plotted as a function of time for three surface intensities of $I_{l}=0.05, I_{2}=0.44$, and $I_{3}=0.90 \mathrm{MWatts} / \mathrm{cm}^{2}$ (Figure 9). This data was obtained using motion analysis software (Kodak Motion Analysis Workstation). The software allows the user to track an image throughout the frames of an exposure, recording its position as 
a function of time. In this measurement, the interface between the first dark region and the first light region was used to estimate the speed of the plume.

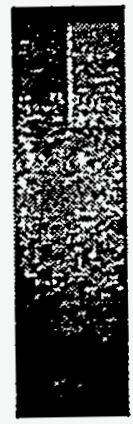

0.66

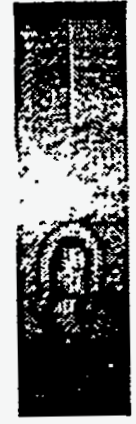

0.83

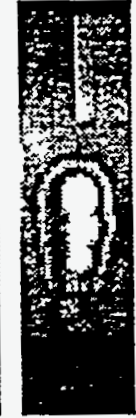

1.00

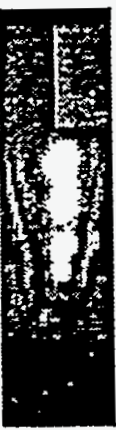

1.16

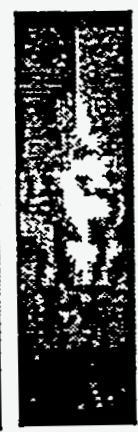

1.33

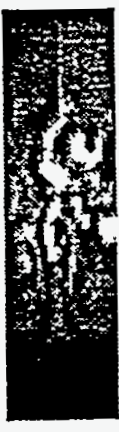

3.50

Figure 8: High-speed laser schlieren images of the plume generated above 304 stainless for an intensity of 0.90 $\mathrm{MW} / \mathrm{cm}^{2}$. The times shown for each frame are in milliseconds.

In each case, a second order polynomial was fit to each set of measurements. Differentiating each polynomial, the following expressions were obtained for the speed of the plume at each intensity,

$$
\begin{aligned}
& z_{1}^{\prime}(t)=1.67-.568 t \\
& z_{2}^{\prime}(t)=1.37-0.018 t \\
& z_{3}^{\prime}(t)=2.64-0.604 t
\end{aligned}
$$

where $z_{\mathrm{n}}{ }^{\prime}$, is the speed of the plume in $\mathrm{cm} / \mathrm{ms}$ at a surface intensity of $I_{n}$ and $t$ is the time in milliseconds. From the above equations, the speed of the plume at $t=1.0 \mathrm{~ms}$ was calculated as $11.0,13.5$, and $20.4 \mathrm{~m} / \mathrm{s}$ at $0.05,0.44$, and $0.90 \mathrm{MWatts} / \mathrm{cm}^{2}$, respectively.

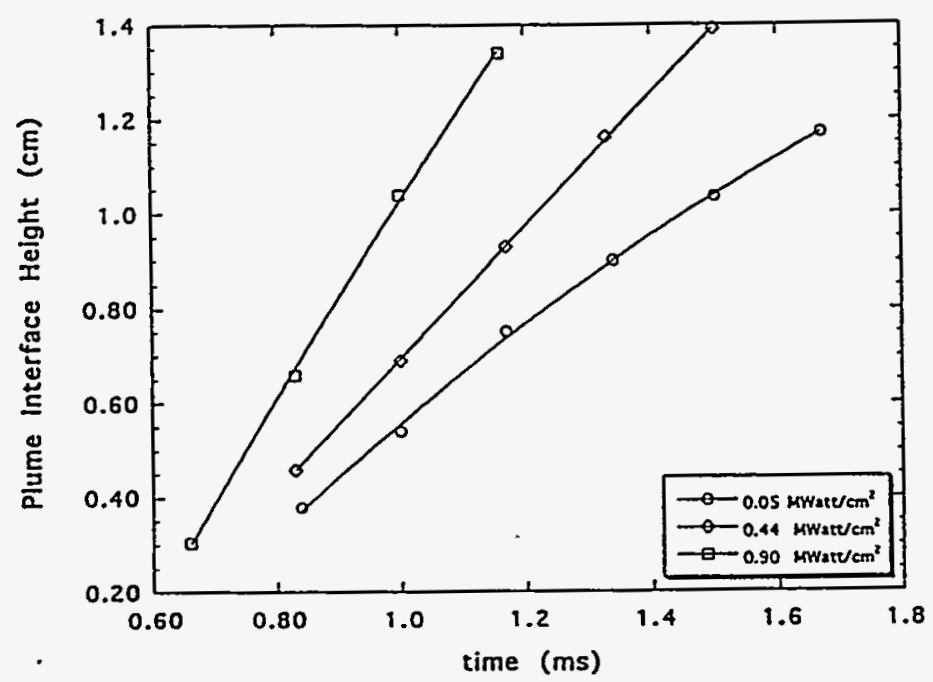

Figure 9: Plot of the plume interface height (measured with respect to the metal surface) vs. time. 


\section{Discussion}

We have shown qualitatively that the plume formed above the metal surface during laser welding forms a highly refractive medium. Refraction is minimal in the central regions of the plume, but increases at increasing distances from the plume axis. As the distance from the plume axis increases, a region exists where zero beam deflection again occurs. This region is then followed by a highly refractive region that forms the edge of the plume. Future work will be performed to relate intensity in the schlieren image to the deflection of the probe laser beam. Finally deflection of the processing laser beam will be determined for a direction of propagation along the plume axis. The measured plume speeds, as well as the observed plume morphology, may be used as input and validation for models of surface vaporization, enhancing the understanding of laser welding.

\section{Conclusions}

The results of this work are summarized as follows:

(1) Laser schlieren imaging has been shown to be a viable method of revealing the refractive properties of a laser-generated plume.

(2) Laser schlieren imaging has shown that the plume of vaporized metal propagates away from the metal surface in the form of a highly refractive region of gas.

(3) The speed of the plume was measured at $t=1.0 \mathrm{~ms}$ as $11.0,13.5$, and $20.4 \mathrm{~m} / \mathrm{s}$ for surface intensities of $0.05,0.44$, and $0.90 \mathrm{MWatts} / \mathrm{cm}^{2}$ respectively.

\section{Acknowledgments}

This work was performed at Sandia National Laboratories supported by the U.S. Department of Energy under contract number DE-AC04-94AL85000. The authors would also like to acknowledge Joseph A. Romero for his technical support.

\section{References}

1 Cieslak, M. J., and Jellison, J. L. (Feb, 1989). A Perspective on Welding Science. MRS Bulletin. XIV. 2: 32-38.

2 Mills, G. S. (1977). Analysis of High Manganese Stainless Steel Weldability Problem.. Welding Journal. 56: 186S - 188S.

3 Peebles, H. C., and Williamson, R. L. (1987). The Role of the Metal Vapor Plume in Pulsed Nd:YAG Laser Welding on Aluminum 1100. Proc. of LAMP, Osaka, Japan, 19-24.

4 Russo, A. (1995). Sandia National Laboratories. Albuquerque, New Mexico.

5 Merzkirch, W. (1974). Flow Visualization. Academic Press, New York. 134-152. 
6 Kogelschatz, U., Schneider, W. R. (1971). A Quantitative Laser Schlieren Technique. 10th International Conference on Phenomena in Ionized Gases. 1. 420.

\section{DISCLAIMER}

This report was prepared as an account of work sponsored by an agency of the United States Government. Neither the United States Government nor any agency thereof, nor any of their employees, makes any warranty, express or implied, or assumes any legal liability or responsibility for the accuracy, completeness, or usefulness of any information, apparatus, product, or process disclosed, or represents that its use would not infringe privately owned rights. Reference herein to any specific commercial product, process, or service by trade name, trademark, manufacturer, or otherwise does not necessarily constitute or imply its endorsement, recommendation, or favoring by the United States Government or any agency thereof. The views and opinions of authors expressed herein do not necessarily state or reflect those of the United States Government or any agency thereof. 